\title{
ON A POINTWISE ERGODIC THEOREM FOR MULTIPARAMETER SEMIGROUPS
}

\author{
RYOTARO SATO
}

Abstract

Let $T_{i}(i=1,2, \ldots, d)$ be commuting null preserving transformations on a finite measure space $(X, \mathcal{F}, \mu)$ and let $1 \leq p<\infty$. In this paper we prove that for every $f \in L_{p}(\mu)$ the averages

$$
A_{n} f(x)=(n+1)^{-d} \sum_{0 \leq n_{i} \leq n} f\left(T_{1}^{n_{1}} T_{2}^{n_{2}} \ldots T_{d}^{n_{d}} x\right)
$$

converge a.e. on $X$ if and only if there exists a finite invariant measure $\nu$ (under the transformations $T_{i}$ ) absolutely continuous with respect to $\mu$ and a sequence $\left\{X_{N}\right\}$ of invariant sets with $X_{N} \uparrow X$ such that $\nu B>0$ for all nonnull invariant sets $B$ and such that the Radon-Nikodym derivative $v=d \nu / d \mu$ satisfies $v \in$ $L_{q}\left(x_{N}, \mu\right), 1 / p+1 / q=1$, for each $N \geq 1$.

\section{Introduction}

We refer to [2] for the basic notation in ergodic theory. Let $(X, \mathcal{F}, \mu)$ be a finite measure space and let $T_{i}: X \rightarrow X(i=1,2, \ldots, d)$ be commuting null preserving transformations, where $d \geq 1$ is a fixed integer. Associated with these transformations $T_{i}$ and for any measurable function $f$ on $X$ we have the averages

$$
A_{n} f(x)=(n+1)^{-d} \sum_{0 \leq n_{i} \leq n} f\left(T_{1}^{n_{1}} T_{2}^{n_{2}} \ldots T_{d}^{n_{d}} x\right) \quad(n \geq 0)
$$

and the maximal operator

$$
M f=\sup _{n \geq 0} A_{n}|f| .
$$


Further each $T_{i}$ defines, by the Radon-Nikodym Theorem, a unique positive linear contraction operator $T_{i}^{*}$ on $L_{1}(\mu)$ by the relation

$$
\int_{B} T_{i}^{*} u d \mu=\int_{T_{i}^{-1} B} u d \mu \quad\left(u \in L_{1}(\mu), B \in \mathcal{F}\right) .
$$

Under the additional hypothesis that all the transformations $T_{i}$ are invertible, Martín-Reyes [3] has recently proved the equivalence of the following conditions, for $1 \leq p<\infty$.

(a) The sequence $\left\{A_{n} f\right\}$ converges a.e. for all $f$ in $L_{p}(\mu)$;

(b) There exists a positive measurable function $U$ on $X$ such that

$$
\int_{\left\{\left|A_{n} f\right|>t\right\}} U d \mu \leq t^{-p} \int_{X}|f|^{p} d \mu \quad\left(t>0, f \in L_{p}(\mu)\right) .
$$

In this paper, without assuming the invertibility hypothesis on the transformations $T_{i}$, we intend to characterize those finite measures $\mu$ for which (a) holds.

\section{The result}

Theorem. Let $(X, \mathcal{F}, \mu)$ be a finite measure space and let $T_{i}: X \rightarrow X$ $(i=1,2, \ldots, d)$ be commuting null preserving transformations. If $1 \leq$ $p<\infty$, then the following are equivalent.

(a) For any $f \in L_{p}(\mu)$ the sequence $\left\{A_{n} f\right\}$ converges to a finite limit a.e. on $X$.

(b) For any $u \in L_{1}(\mu)$ the averages

$$
A_{n}^{*} u=(n+1)^{-d} \sum_{0 \leq n_{i} \leq n} T_{1}^{*^{n_{1}}} T_{2}^{*^{n_{2}}} \ldots T_{d}^{*^{n} d} u \quad(n \geq 0)
$$

converge a.e. on $X$ and also in the norm topology of $L_{1}(\mu)$; further to every $v \in L_{1}^{+}(\mu)$ with $T_{i}^{*} v=v$ for all $i=1,2, \ldots, d$ there corresponds a sequence $\left\{X_{N}\right\}$ of invariant sets with $X_{N} \uparrow X$ such that $v \in L_{q}\left(X_{N}, \mu\right), 1 / p+1 / q=1$, for all $N \geq 1$.

(c) There exists $v \in L_{1}^{+}(\mu)$ with $T_{i}^{*} v=v$ for all $i=1,2, \ldots, d$ and $a$ sequence $\left\{X_{N}\right\}$ of invariant sets with $X_{N} \uparrow X$ such that $\int_{B} v d \mu>$ 0 for all nonnull invariant sets $B$ and such that $v \in L_{q}\left(X_{N}, \mu\right)$, $1 / p+1 / q=1$, for all $N \geq 1$.

(d) $M f<\infty$ for all $f \in L_{p}(\mu)$. 
(e) There exists a positive measurable function $U$ on $X$ such that

$$
\int_{\{M f>t\}} U d \mu \leq t^{-p} \int_{X}|f|^{p} d \mu \quad\left(t>0, f \in L_{p}(\mu)\right) .
$$

(f) There exists a positive measurable function $U$ on $X$, a constant $r>0$, and a subsequence $\{n(k)\}$ of $\{n\}$ such that

$$
\int_{\left\{\left|A_{n(k)} f\right|>t\right\}} U d \mu \leq t^{-r}\left(\int_{X}|f|^{p} d \mu\right)^{r / p} \quad\left(t>0, f \in L_{p}(\mu)\right) .
$$

We begin by proving the following lemma, which deals with the case $p=\infty$.

Lemma. Let $(X, \mathcal{F}, \mu)$ be a finite measure space and let $T_{i}: X \rightarrow X$ $(i=1,2, \ldots, d)$ be commuting null preserving transformations. Then the following are equivalent.

(a) The sequence $\left\{A_{n} f\right\}$ converges a.e. on $X$ for all $f \in L_{\infty}(\mu)$.

(b) The sequence $\left\{A_{n}^{*} u\right\}$ converges in the norm topology of $L_{1}(\mu)$ for all $u \in L_{1}(\mu)$.

(c) For any $u \in L_{1}^{+}(\mu)$ with $\|u\|_{1}>0$ the pointwise limit $u_{0}^{*}(x)=$ $\lim _{n} A_{n}^{*}(x)$ exists a.e. on $X$ and satisfies $\left\|u_{0}^{*}\right\|_{1}>0$.

(d) For any $u \in L_{1}^{+}(\mu)$ with $\|u\|_{1}>0$ we have

$$
0<\left\|\liminf _{n} A_{n}^{*} u\right\|_{1}<\infty .
$$

Proof:

(a) $\Rightarrow$ (b) follows from a mean ergodic theorem (see e.g. [2, Theorem 2.1.5]).

(b) $\Rightarrow$ (a) and (c). Let $v_{0}=$ strong- $\lim _{n} A_{n}^{*} 1\left(\in L_{1}^{+}(\mu)\right)$. Since $T_{i}^{*} v_{0}=v_{0}$ for all $i=1,2, \ldots, d$, we have

$$
Y \subset T_{i}^{-1} Y \text { for all } i=1,2, \ldots, d \text {, where } Y=\left\{v_{0}>0\right\} .
$$

Since the measure $\nu=v_{0} d \mu$ is invariant under the $T_{i}$ 's, we may regard the transformations $T_{i}$ as commuting measure preserving transformations on a finite measure space $\left(Y, v_{0} d \mu\right)$. Then, by the classical multiparameter pointwise ergodic theorem, for any $f \in L_{\infty}(\mu)$ the sequence 
$\left\{A_{n} f\right\}$ converges a.e. on $Y$. To prove the a.e. convergence of $\left\{A_{n} f\right\}$ on $X \backslash Y$, it is sufficient to show that

$$
\left(T_{1} T_{2} \ldots T_{d}\right)^{-n} Y \uparrow X
$$

To do this, let $B=\lim _{n}\left(T_{1} T_{2} \ldots T_{d}\right)^{-n} Y$. We see easily that $T_{i}^{-1} B=B$ for all $i=1,2, \ldots, d$, i.e., $B$ is an invariant set. Hence

$$
\mu(X \backslash B)=\int_{X \backslash B} A_{n}^{*} 1 d \mu \rightarrow \int_{X \backslash B} v_{0} d \mu=\int_{\left\{v_{0}=0\right\}} v_{0} d \mu=0 .
$$

To prove (c), let $u \in L_{1}^{+}(\mu)$ and $\|u\|_{1}>0$. Since $\left\|A_{n}^{*} u\right\|_{1}=\|u\|_{1}>$ 0 and $\left\{A_{n}^{*} u\right\}$ converges in the norm topology of $L_{1}(\mu)$, it is sufficient to prove the a.e. convergence of $\left\{A_{n}^{*} u\right\}$. Since the transformations $T_{i}$ preserve the measure $\nu=v_{0} d \mu$, the classical pointwise ergodic theorem for multiparameter semigroups of Dunford-Schwartz operators and an approximation argument imply that $\left\{A_{n}^{*} u\right\}$ converges a.e. on $Y$.

To prove that $\lim _{n} A_{n}^{*} u(x)=0$ a.e. on $X \backslash Y$, we use Brunel's Theorem (see e.g. [2, Theorem 6.3.4]) concerning an ergodic inequality for commuting linear contraction operators on $L_{1}(\mu)$ : there exists a constant $K_{d}>0$ and a positive linear operator $Q$ on $L_{\infty}(\mu)$ of the form

$$
Q f(x)=\sum_{n_{i} \geq 0} a\left(n_{1}, n_{2}, \ldots, n_{d}\right) f\left(T_{1}^{n_{1}} T_{2}^{n_{2}} \ldots T_{d}^{n_{d}} x\right),
$$

where $a\left(n_{1}, n_{2}, \ldots, n_{d}\right)>0$ and $\sum_{n_{i} \geq 0} a\left(n_{1}, n_{2}, \ldots, n_{d}\right)=1$, such that if $Q^{*}$ denotes the positive linear operator on $L_{1}(\mu)$ associated with $Q$, then

$$
\underset{n}{\limsup } A_{n}^{*} u \leq K_{d} \cdot \underset{n}{\limsup }(n+1)^{-1} \sum_{i=0}^{n} Q^{* i} u \quad\left(u \in L_{1}^{+}(\mu)\right) .
$$

Let $C=\left\{x: \sum_{i=0}^{\infty} Q^{* i} u=\infty\right\} \backslash Y$. Since $\left\|Q^{*}\right\|_{1}=1$, it follows that $Q 1_{C} \geq 1_{C}, 1_{C}$ being the indicator function of $C$. Thus we have $C \subset$ $T_{i}^{-1} C$ for all $i=1,2, \ldots, d$, and hence

$$
\mu C \leq \int_{X} A_{n} 1_{C} d \mu=\int_{C} A_{n}^{*} 1 d \mu \rightarrow \int_{C} v_{0} d \mu=\int_{\left\{v_{0}=0\right\}} v_{0} d \mu=0 .
$$

This proves that $\lim _{n} A_{n}^{*} u(x)=0$ a.e. on $X \backslash Y$.

(c) $\Rightarrow$ (d). Obvious. 
(d) $\Rightarrow$ (b). There exists $v_{0} \in L_{1}^{+}(\mu)$ with $T_{i}^{*} v_{0}=v_{0}$ for all $i=$ $1,2, \ldots, d$ such that if $v \in L_{1}^{+}(\mu)$ satisfies $T_{i}^{*} v=v$ for all $i=1,2, \ldots, d$ then $\{v>0\} \subset\left\{v_{0}>0\right\}$. Let $Y=\left\{v_{0}>0\right\}$ and

$$
B=\lim _{n}\left(T_{1} T_{2} \ldots T_{d}\right)^{-n} Y .
$$

Since $B$ and $X \backslash B$ are invariant sets, it follows that if $u \in L_{1}^{+}(X \backslash B, \mu)$ and $\|u\|_{1}>0$ then the function

$$
\tilde{u}_{0}=\liminf _{n} A_{n}^{*} u \text { satisfies } \quad\left\{\tilde{u}_{0}>0\right\} \cap\left\{v_{0}>0\right\}=\phi .
$$

But this is impossible, since $T_{i}^{*} \tilde{u}_{0}=\tilde{u}_{0} \in L_{1}^{+}(\mu)$ for all $i=1,2, \ldots, d$ and (d) implies that $\mu\left\{\tilde{u}_{0}>0\right\}>0$. We conclude that

$$
\left(T_{1} T_{2} \ldots T_{d}\right)^{-n} Y \uparrow X .
$$

Hence by an approximation argument we see that $\left\{A_{n}^{*} u\right\}$ converges in the norm topology of $L_{1}(\mu)$ for all $u \in L_{1}(\mu)$, completing the proof.

Proof of the Theorem: (a) $\Rightarrow$ (b). The first part of (b) follows from the lemma. To prove the second part, let $v \in L_{1}^{+}(\mu)$ be such that $T_{i}^{*} v=v$ for all $i=1,2, \ldots, d$. Putting $Y=\{v>0\}$, we see that the transformations $T_{i}$ can be regarded as commuting null preserving transformations on the measure space $(Y, \mu)$. Since the measure $\nu=v d \mu$ is invariant under the transformations $T_{i}$, it follows that these $T_{i}$ are conservative on $(Y, \mu)$.

By this and the fact that for each $f$ in $L_{p}(Y, \mu)$ the sequence $\left\{A_{n} f\right\}$ converges to a finite limit a.e. on $Y$, we can apply Theorem 3.1 in [4] to infer that there exists a sequence $\left\{Y_{N}\right\}$ of sets in $\mathcal{I}_{Y}$, where

$$
\mathcal{I}_{Y}=\left\{B \in \mathcal{F}: B \subset Y, B=Y \cap T_{i}^{-1} B \text { for all } i=1,2, \ldots, d\right\},
$$

such that $Y_{N} \uparrow Y$ and $v \in L_{q}\left(Y_{N}, \mu\right)$ for all $N \geq 1$. Then, letting

$$
X_{N}=\left[\lim _{n}\left(T_{1} T_{2} \ldots T_{d}\right)^{-n} Y_{N}\right] \cup\left[X \backslash \lim _{n}\left(T_{1} T_{2} \ldots T_{d}\right)^{-n} Y\right],
$$

we have $v \in L_{q}\left(X_{N}, \mu\right)$ for all $N \geq 1, X_{N} \uparrow X$, and $X_{N} \in \mathcal{I}$ where

$$
\mathcal{I}=\left\{B \in \mathcal{F}: B=T_{i}^{-1} B \text { for all } i=1,2, \ldots, d\right\} .
$$

(b) $\Rightarrow$ (c). It is enough to put $v=$ strong- $\lim _{n} A_{n}^{*} 1$. that

(c) $\Rightarrow$ (a). Put $Y=\{v>0\}$. It follows (cf. the proof of the lemma)

$$
\left(T_{1} T_{2} \ldots T_{d}\right)^{-n} Y \uparrow X
$$


Hence it is sufficient to prove that for each $f$ in $L_{p}(Y, \mu)$ the sequence $\left\{A_{n} f\right\}$ converges to a finite limit a.e. on $Y$; this follows from the equivalence of (a) and (f) of Theorem 3.1 in [4], since the transformations $T_{i}$ may be regarded as commuting conservative null preserving transformations on the measure space $(Y, \mu)$.

(a) $\Rightarrow$ (d). Obvious.

(d) $\Rightarrow$ (e). This follows from Nikishin's Theorem (see e.g. [1, p. 536])

(e) $\Rightarrow$ (f). Obvious.

(f) $\Rightarrow$ (a). We may suppose that $0<U \leq 1$ on $X$. Using an approximation argument we see (cf. the proof of (d) $\Rightarrow$ (a) of Theorem 3.1 in [4]) that

$$
\lim _{\mu B \rightarrow 0} \sup _{k \geq 1} \int_{B} A_{n(k)}^{*} 1 d \mu=0 .
$$

Hence by a mean ergodic theorem we see that the sequence $\left\{A_{n}^{*} 1\right\}$ converges in the norm topology of $L_{1}(\mu)$. Write $v=$ strong- $\lim _{n} A_{n}^{*} 1$ and $Y=$ $\{v>0\}$. Since $T_{i}^{*} v=v$ for all $i=1,2, \ldots, d$ and $\left(T_{1} T_{2} \ldots T_{d}\right)^{-n} Y \uparrow X$, it follows from the classical multiparameter pointwise ergodic theorem that for any $f \in L_{p}^{+}(\mu)$ the limit

$$
f^{*}(x)=\lim _{n} A_{n} f(x)
$$

exists a.e. on $X$ (but may be equal to infinity on some subset of $X$ ).

To prove that $f^{*}<\infty$ a.e. on $X$, we observe that $\left\{f^{*}=\infty\right\} \subset$ $\liminf _{k}\left\{A_{n(k)} f>t\right\}$ for all $t>0$; hence by Fatou's Lemma and $(f)$

$$
\int_{\left\{f^{*}=\infty\right\}} U d \mu \leq \liminf _{k} \int_{\left\{A_{n(k)} f>t\right\}} U d \mu \leq t^{-r}\left(\int_{X} f^{p} d \mu\right)^{r / p} .
$$

Letting $t \uparrow \infty$, we have $\int_{\left\{f^{*}=\infty\right\}} U d \mu=0$ and $\mu\left\{f^{*}=\infty\right\}=0$. The proof is complete.

Since the above proofs of the implications (a) $\Rightarrow$ (d) $\Rightarrow$ (e) $\Rightarrow$ (f) $\Rightarrow$ (a) hold for $0<p<\infty$, we have the

Corollary. Let $(X, \mathcal{F}, \mu)$ be a finite measure space and let $T_{i}: X \rightarrow$ $X(i=1,2, \ldots, d)$ be commuting null preserving transformations. Let $0<p, r<\infty$. If there exists a subsequence $\{n(k)\}$ of $\{n\}$ such that the operators $A_{n(k)}$ are equicontinuous mappings from $L_{p}(\mu)$ to $L_{r}(\mu)$ then 
for any $f \in L_{p}(\mu)$ the sequence $\left\{A_{n} f\right\}$ converges to a finite limit a.e. on $X$.

Proof: This follows from the equivalence of (a) and (f) of the theorem.

\section{References}

1. J. García-Cuerva and J. L. Rubio de Francia, "Weighted Norm Inequalities and Related Topics," North Holland, Amsterdam, 1985.

2. U. Krengel, "Ergodic Theorems," Walter de Gruyter, Berlin, 1985.

3. F. J. MARTín-REYes, On a pointwise ergodic theorem for multiparameter groups, in "Almost Everywhere Convergence," (G. A. Edgar and L. Sucheston, editors), Academic Press, Boston, 1989, pp. $267-279$.

4. R. SATo, Multiparameter pointwise ergodic theorems for Markov operators on $L_{\infty}$, submitted for publication.

Department of Mathematics

School of Science

Okayama University

Okayama 700

JAPAN

Rebut el 5 de Juliol de 1993

Added in proof: An obvious argument shows that condition (c) of the theorem may be sharpened as follows. (c') There exists $v \in L_{q}^{+}(\mu)$ with $T_{i}^{*} v=v$ for all $1 \leq i \leq d$ such that $\int_{B} v d \mu>0$ for all nonnull invariant sets $B$. 